\title{
Cardiovascular risk may be increased in women with unexplained infertility
}

\author{
Fatma Ferda Verit' ${ }^{1}$, Fadile Yildiz Zeyrek ${ }^{2}$, Ali Galip Zebitay ${ }^{1}$, Hurkan Akyol ${ }^{1}$ \\ ${ }^{1}$ Department of Obstetrics and Gynecology, Suleymaniye Maternity, Research, and Training Hospital, Istanbul; ${ }^{2}$ Department of Microbiology, Harran \\ University Faculty of Medicine, Sanliurfa, Turkey
}

Objective: Growing evidence suggests that increased cardiovascular disease (CVD) risk is associated with female infertility caused by conditions such as polycystic ovarian disease, obesity, thyroid dysfunction, and endometriosis. The aim of this study was to evaluate whether any relationship exists between CVD and unexplained infertility.

Methods: Sixty-five women with unexplained infertility and 65 fertile controls were enrolled in the study. CVD risk markers such as low-density lipoprotein (LDL), high-density lipoprotein (HDL), total cholesterol (TC), triglycerides (TG), insulin resistance (defined by the homeostasis model assessment ratio), and high-sensitivity C-reactive protein (hs-CRP) were assessed.

Results: TG, TC, LDL, and hs-CRP levels were higher and HDL levels were lower in patients with unexplained infertility than in fertile controls ( $p<0.05$ for all). Positive associations were found between unexplained infertility and TG, TC, LDL, and hs-CRP levels, and a negative correlation was found for HDL ( $p<0.05$ for all). Multivariate logistic regression analysis showed that TG, HDL, and hs-CRP were independent variables associated with unexplained infertility.

Conclusion: Our study showed that women with unexplained infertility had an atherogenic lipid profile and elevated hs-CRP levels, suggesting a higher risk of developing CVD in the future. Further studies with larger groups are needed to investigate the nature of this link.

Keywords: Cardiovascular diseases; C-reactive protein; Dyslipidemia; Infertility female; Insulin resistance; Risk factors

\section{Introduction}

Unexplained infertility refers to the $30 \%$ to $40 \%$ of infertile couples in whom standard investigations, including semen analysis and tests of ovulation and tubal patency, cannot detect any major abnormality $[1,2]$. Couples with unexplained infertility suffer from difficulties in fecundity. The possible underlying etiologies are diminished ovarian reserve, advanced age, minimal and mild endometriosis, endocrine abnormalities, oxidative stress, and defective endometrial receptivity [3].

Cardiovascular disease (CVD) is the one of the most common causes

Received: Jun 29, 2016 · Revised: Nov 15, 2016 · Accepted: Nov 21, 2016 Corresponding author: Fatma Ferda Verit

Department of Obstetrics and Gynecology, Suleymaniye Maternity, Research,and Training Hospital, Zeytinburnu, Istanbul 34020, Turkey Tel: +90-212-664-5355 Fax:+90-212-664-5357 E-mail:fverit@gmail.com

This is an Open Access article distributed under the terms of the Creative Commons Attribution Non-Commercial License (http://creativecommons.org/licenses/by-nc/4.0/) which permits unrestricted non-commercial use, distribution, and reproduction in any medium, provided the original work is properly cited. of morbidity and mortality in the world, and atherosclerosis is known to be the main reason for increased cardiovascular risk. It is widely known that inflammation plays an important role in atherosclerosis [4]. Inflammatory factors, such as monocyte/macrophage colonystimulating factor, transforming growth factor- $\beta 1$, interleukin- 6 and C-reactive protein (CRP) have been shown to be involved in atherogenesis [5].

High-sensitivity CRP (hs-CRP) is a marker of chronic low grade inflammation that is increased in patients with unstable angina and is a biochemical risk factor for myocardial infarction in healthy individuals [6]. It is the strongest predictor of the risk of cardiovascular events among 12 baseline laboratory markers, including lipids and homocysteine [7]. An elevated hs-CRP level is also associated with metabolic syndrome parameters such as insulin resistance, abdominal obesity, and dyslipidemia [8].

Insulin resistance is an important cofactor in the development and the progression of CVD [9]. It is associated with obesity and dyslipidemia, and is a component of metabolic syndrome [10]. It is also asso- 
ciated with low-grade chronic inflammation [11]. Cardiovascular insulin resistance leads to endothelial dysfunction, impaired cardiac diastolic relaxation, vascular relaxation, decreased coronary blood flow, and increased susceptibility to ischemia [12].

It has been reported that infertility may share some common pathways with CVD [13]. Polycystic ovarian syndrome (PCOS), thyroid dysfunction, and obesity are all known to be associated with CVD [13]. Hypercoagulable states or thrombophilia may contribute to early miscarriages, a potential unrecognized cause of subfertility [14]. Women with subfertility have also increased levels of psychological stress, as manifested in conditions such as depression and anxiety, which may contribute to CVD [15]. Moreover, oxidative stress, which has an important role in the development of CVD, is also increased in infertile patients with conditions such as endometriosis, PCOS, obesity, and unexplained infertility [16]. However, the association between unexplained infertility and CVD has not been studied in detail.

The aim of this study was to investigate whether any relationship was present between unexplained female infertility and CVD, as assessed by cardiovascular risk markers such as insulin resistance, hsCRP, and dyslipidemia.

\section{Methods}

This study included a total of 130 women aged between 20 and 35 years who were admitted to the Harran University Faculty of Medicine, Gynecology and Obstetrics Clinic. between January 2011 and January 2012. Sixty-five of the subjects were patients with unexplained infertility and 65 were fertile controls. Institutional review board approval and informed consent from all participants were obtained. All infertile women had normal serum concentrations of follicle-stimulating hormone (FSH) on day 3 of their menstrual cycles, normal estradiol levels, normal hysterosalpingograms indicating a lack of tubal occlusion, and normal ovaries and uterus detected by transvaginal ultrasonography and/or hysterosalpingography. Their partners had normal semen analysis results. Fertility was defined as initiation of a successful pregnancy within the last 12 months before the participation of the study.

Additional inclusion criteria were serum FSH levels within normal limits (1-10 IU/L), spontaneous menses or a positive bleeding response to progestogen withdrawal, and normal serum prolactin and thyroid-stimulating hormone levels. The primary exclusion criteria were hypothalamic amenorrhea; premature ovarian failure; congenital adrenal hyperplasia; ovarian cysts; androgen-secreting tumors or ovarian tumors; Cushing syndrome; CVD; PCOS; diabetes mellitus; insulin resistance; metabolic syndrome; hyperlipidemia; a history of major depression; the use of any psychoactive medications, cocaine, or opiates; age above 35 years; the presence of neoplastic, infectious, autoimmune, liver, or kidney diseases; smoking; and alcohol consumption. None of the patients had previously been diagnosed with endometriosis or had any clinical symptoms thereof.

Insulin resistance was estimated by the homeostasis model assessment of insulin resistance (HOMA-IR) using the following formula: fasting blood sugar $(\mathrm{mg} / \mathrm{dL}) \times$ fasting insulin $(\mu \mathrm{lU} / \mathrm{mL}) / 405$ [17].

All participants underwent a standardized initial clinical, sonographic, and endocrine screening. The clinical screening included age, cycle history, body mass index, and previous medications and/ or surgery. The endocrine screening included serum assays for glucose, insulin, prolactin, FSH, luteinizing hormone (LH), thyroid-stimulating hormone, estradiol, progesterone, free testosterone, total testosterone, sex hormone-binding globulin, and dehydroepiandrosterone sulfate. Studies were performed between menstrual cycle days 3 and 5 . Fasting venous blood samples were taken between 8:00 and 10:00 AM after a 12-hour overnight fast. Blood samples were then centrifuged within 2 hours and assessed on the same day.

\section{Measurement of hs-CRP levels}

The serum level of hs-CRP was measured immediately with an Aeroset autoanalyzer (Abbott, Maidenhead, UK) using a spectrophotometric commercial kit (Scil Diagnostics GmbH, Viernheim, Germany).

\section{Other parameters}

Levels of triglycerides (TG), total cholesterol (TC), high-density lipoprotein $(\mathrm{HDL})$, and low-density lipoprotein $(\mathrm{LDL})$ were determined using commercially available assay kits (Abbott) with an autoanalyzer (Aeroset, Abbott).

\section{Statistical analysis}

Baseline characteristics of the groups are shown as the mean \pm standard deviation. Variables with a skewed distribution were logtransformed for all analyses. Laboratory and anthropometric parameters of patients were compared using the Student'st-test. Correlations between unexplained infertility and hs-CRP, TG, TC, LDL, and HDL were assessed using the Spearman rank test. In addition, multivariate logistic regression analysis was applied to analyze the associations between cardiovascular risk markers and unexplained infertility. The $p$-values $<0.05$ were considered to indicate statistical significance.

\section{Results}

The baseline characteristics of the patients are summarized in Table 1. No significant differences were found in age, body mass index, $\mathrm{FSH}$, LH, estradiol, fasting glucose, insulin, or HOMA-IR levels between the groups. Women with unexplained infertility had significantly higher levels of TG, TC, LDL, and hs-CRP, and lower HDL levels 
Table 1. Baseline characteristics of the patients

\begin{tabular}{lccc}
\hline Variable & Women with unexplained infertility $(\mathrm{n}=65)$ & Fertile controls $(\mathrm{n}=65)$ & $p$-value \\
\hline Age $(\mathrm{yr})$ & $27.5 \pm 2.9$ & $27.6 \pm 3.1$ & 0.93 \\
$\mathrm{BMI}\left(\mathrm{kg} / \mathrm{m}^{2}\right)$ & $24.6 \pm 2.3$ & $24.9 \pm 2.3$ & 0.49 \\
FSH $(\mathrm{mlU} / \mathrm{mL})$ & $6.8 \pm 1.1$ & $6.6 \pm 1.2$ & 0.28 \\
LH $(\mathrm{mlU} / \mathrm{mL})$ & $4.8 \pm 1.1$ & $4.6 \pm 1.1$ & 0.40 \\
Estradiol $(\mathrm{pg} / \mathrm{mL})$ & $57.4 \pm 10.8$ & $55.4 \pm 10.6$ & 0.29 \\
TG $(\mathrm{mg} / \mathrm{dL})$ & $181.1 \pm 33.2$ & $122.4 \pm 32.4$ & $<0.001$ \\
TC $(\mathrm{mg} / \mathrm{dL})$ & $202.0 \pm 30.4$ & $176.0 \pm 29.9$ & $<0.001$ \\
HDL $(\mathrm{mg} / \mathrm{dL})$ & $42.4 \pm 11.3$ & $53.6 \pm 12.3$ & $<0.001$ \\
LDL $(\mathrm{mg} / \mathrm{dL})$ & $125.3 \pm 32.7$ & $99.1 \pm 31.0$ & $<0.001$ \\
Fasting glucose $(\mathrm{mg} / \mathrm{dL})$ & $80.6 \pm 12.2$ & $78.4 \pm 12.1$ & 0.30 \\
Fasting insulin $(\mu \mathrm{lU} / \mathrm{mL})$ & $5.0 \pm 2.0$ & $4.8 \pm 1.7$ & 0.57 \\
HOMA-IR & $0.9 \pm 0.4$ & $0.9 \pm 0.3$ & 0.35 \\
hs-CRP $(\mathrm{mg} / \mathrm{L})$ & $0.07 \pm 0.0$ & $0.01 \pm 0.0$ & $<0.001$ \\
\hline
\end{tabular}

Values are presented as mean \pm standard deviation.

BMI, body mass index; FSH, follicle-stimulating hormone; LH, luteinizing hormone; TG, triglycerides; $T C$, total cholesterol; HDL, high-density lipoprotein; LDL, low-density lipoprotein; HOMA-IR, homeostasis model assessment of insulin resistance; hs-CRP, high-sensitivity C-reactive protein.

Table 2. Correlations of unexplained infertility with dyslipidemia and hs-CRP

\begin{tabular}{lcc}
\hline Variable & Correlation coefficient & $p$-value \\
\hline $\mathrm{TG}(\mathrm{mg} / \mathrm{dL})$ & 0.68 & $<0.001$ \\
$\mathrm{TC}(\mathrm{mg} / \mathrm{dL})$ & 0.36 & $<0.001$ \\
$\mathrm{HDL}(\mathrm{mg} / \mathrm{dL})$ & -0.44 & $<0.001$ \\
$\mathrm{LDL}(\mathrm{mg} / \mathrm{dL})$ & 0.37 & $<0.001$ \\
$\mathrm{hs}-\mathrm{CRP}(\mathrm{mg} / \mathrm{L})$ & 0.53 & $<0.001$ \\
\hline
\end{tabular}

hs-CRP, high-sensitivity C-reactive protein; TG, triglycerides; TC, total cholesterol; HDL, high-density lipoprotein; LDL, low-density lipoprotein.

than the fertile controls $(p<0.001)$.

Positive associations were found between unexplained infertility and TG $(r=0.68, p<0.001), \mathrm{TC}(r=0.36, p<0.001)$, LDL $(r=0.37$, $p<0.001)$, hs-CRP $(r=0.53, p<0.001)$, and a negative association was found with $\mathrm{HDL}(r=-0.44, p<0.001)$ (Table 2$)$.

Multivariate logistic regression analysis was used to study the relationship between unexplained infertility and dyslipidemia and hsCRP. TG (SE $\beta=0.48, p<0.001)$, HDL (SE $\beta=-0.21, p=0.006)$, and hsCRP (SE $\beta=0.28, p<0.001)$ were independent predictors associated with unexplained infertility. The adjusted $R^{2}$ was 0.70 in the study (Table 3).

\section{Discussion}

In this study, we found that TG, TC, LDL, and hs-CRP levels were elevated and HDL was decreased in women with unexplained infertility. Positive relationships were found between unexplained infertility and hs-CRP, TG, TC, and LDL, and a negative relationship was found with HDL. Of these markers, TG, HDL, and hs-CRP were independent
Table 3. The relationship of unexplained infertility with dyslipidemia and hs-CRP, as assessed by multiple regression analysis

\begin{tabular}{lrrr}
\hline Variable & SE $\beta$ & $t$ & $p$-value \\
\hline TG $(\mathrm{mg} / \mathrm{dL})$ & 0.48 & 6.92 & $<0.001$ \\
HDL $(\mathrm{mg} / \mathrm{dL})$ & -0.21 & -2.80 & 0.006 \\
$\mathrm{hs}-\mathrm{CRP}(\mathrm{mg} / \mathrm{L})$ & 0.28 & 4.68 & $<0.001$ \\
\hline
\end{tabular}

hs-CRP, high-sensitivity C-reactive protein; TG, triglycerides; HDL, high-density lipoprotein.

predictors associated with unexplained infertility.

It is widely known that infertility has been suggested to be a risk factor for CVD [13]. Nulliparity and primiparity have been associated with increased CVD risk in comparison with multiparity $[18,19]$. Many studies have reported a close relationship between menstrual irregularities and future CVD risk [13,20]. A 50\% increased risk of myocardial infarction or coronary heart disease was found among women with menstrual irregularities in comparison with those who had normal cycles from the ages of 20 to 35 years [13]. Obesity, insulin resistance, and hypothyroidism are also related with infertility [13], anovulation, and CVD [21,22]. Another study demonstrated that women with ovarian infertility were at high risk of hypertension from 45 years of age, hypercholesterolemia at all ages, and diabetes mellitus before 45 years of age [23]. Early miscarriages, which may be an etiological factor of childlessness, can result from coagulation disorders or thrombophilia $[14,24]$. Hypercoagulable states are associated with CVD risk [25]. Furthermore, coagulation defects may affect the development of subclinical atherosclerosis and plaque formation [26].

Advanced maternal age, minimal to mild endometriosis, endocrine abnormalities, diminished ovarian reserve, and oxidative stress have been identified as possible etiological factors for unexplained infertil- 
ity. It is widely known that CVD increases with age. Endometriosis, another common cause of unexplained infertility, is characterized by local and systemic chronic inflammation, dyslipidemia, and oxidative stress [27]. In addition, endometriosis has been reported to be associated with subclinical atherosclerosis [28]. Recently, endothelial dysfunction, which is accepted as early event of atherosclerosis and leads to structural atherosclerotic changes in the vascular wall, has been identified in women with endometriosis [29]. CVD risk factors were found to be increased in patients with diminished ovarian reserve [30,31]. Many studies have shown that aging, obesity, dyslipidemia, and chronic inflammation are related with reduced oocyte quality and quantity [30,32,33].

Strong evidence suggests that CVD is linked with oxidative stress. Oxidative stress also plays an important role in the etiology of unexplained infertility [16]. Reactive oxygen species can negatively affect ovulation, fertilization, and embryo quality [16]. Pregnancy and implantation rates have been found to be lower and abortion rates to be higher in women with elevated oxidative stress [34]. Although many studies have shown a link between oxidative stress and unexplained infertility, no previous study has evaluated the possible association between cardiovascular risk markers, such as dyslipidemia and hs-CRP, and unexplained infertility. For that reason, it is notable that in this study we determined that cardiovascular risk markers were increased in these patients. Moreover, a close relationship was found between these markers and unexplained infertility.

Infertile patients also have high levels of psychological stress, reflected in conditions such as depression and anxiety. This chronic stress has been known to adversely affect pregnancy and live birth rates [35]. Depression and anxiety may also alter immunomodulation and lead to vascular dysfunction, thereby contributing to CVD [36].

Insulin resistance and chronic low-grade inflammation are associated with cardiovascular risk, and these parameters can be used as CVD markers, as shown by many studies $[6,7,9]$. Both of those markers induce many changes in blood lipid levels, leading to a proatherogenic lipid profile [37,38]. However, we did not find any differences in terms of fasting glucose, insulin, or HOMA-IR levels between the groups. This may be because the unexplained infertility group involved many etiological factors with varying pathophysiology, such as minimal to mild endometriosis, aging, diminished ovarian reserve, endocrine abnormalities, oxidative stress, and defective endometrial receptivity.

TG, hs-CRP, and HDL were independent variables correlated with unexplained infertility. The identification of these parameters as independent variables strengthens the hypothesis of our study.

Our study had some limitations. First, the study population was small and hospital-based. Second, undiagnosed mild endometriosis may have been included in this study. Third, we did not study direct markers of atherosclerosis, such as endothelial dysfunction and carotid intima thickness. Another limitation is that we could not exclude the possible presence of other potential confounding factors, such as family history and differences in diet, smoking, and/or physical activity patterns.

In conclusion, we found that CVD risk may be increased in women with unexplained infertility. A cardiovascular consultation and lifestyle changes may be warranted in these patients. Further studies with larger groups are needed to investigate the nature of this link.

\section{Conflict of interest}

No potential conflict of interest relevant to this article was reported.

\section{References}

1. Smith S, Pfeifer SM, Collins JA. Diagnosis and management of female infertility. JAMA 2003;290:1767-70.

2. Crosignani PG, Collins J, Cooke ID, Diczfalusy E, Rubin B. Recommendations of the ESHRE workshop on 'Unexplained Infertility': Anacapri, August 28-9, 1992. Hum Reprod 1993;8:977-80.

3. Siristatidis C, Bhattacharya S. Unexplained infertility: does it really exist? Does it matter? Hum Reprod 2007;22:2084-7.

4. Bevc S, Sabic S, Hojs R. Atherosclerosis in hemodialysis patients: the role of microinflammation. Ren Fail 2008;30:1012-6.

5. Ikonomidis I, Stamatelopoulos K, Lekakis J, Vamvakou GD, Kremastinos DT. Inflammatory and non-invasive vascular markers: the multimarker approach for risk stratification in coronary artery disease. Atherosclerosis 2008;199:3-11.

6. Hansson GK. Atherosclerosis-an immune disease: the Anitschkov lecture 2007. Atherosclerosis 2009;202:2-10.

7. Kelly CC, Lyall H, Petrie JR, Gould GW, Connell JM, Sattar N. Low grade chronic inflammation in women with polycystic ovarian syndrome. J Clin Endocrinol Metab 2001;86:2453-5.

8. Frohlich M, Imhof A, Berg G, Hutchinson WL, Pepys MB, Boeing H, et al. Association between $C$-reactive protein and features of the metabolic syndrome: a population-based study. Diabetes Care 2000;23:1835-9.

9. Despres JP, Lamarche B, Mauriege P, Cantin B, Dagenais GR, Moorjani $S$, et al. Hyperinsulinemia as an independent risk factor for ischemic heart disease. N Engl J Med 1996;334:952-7.

10. Farin HM, Abbasi F, Kim SH, Lamendola C, McLaughlin T, Reaven GM. The relationship between insulin resistance and dyslipidaemia in cigarette smokers. Diabetes Obes Metab 2007;9:65-9.

11. Kemp DM. Does chronic low-grade endotoxemia define susceptibility of obese humans to insulin resistance via dietary effects 
on gut microbiota? Adipocyte 2013;2:188-90.

12. Aroor A, McKarns S, Nistala R, Demarco V, Gardner M, GarciaTouza M, et al. DPP-4 inhibitors as therapeutic modulators of immune cell function and associated cardiovascular and renal insulin resistance in obesity and diabetes. Cardiorenal Med 2013;3: 48-56.

13. Parikh NI, Cnattingius S, Mittleman MA, Ludvigsson JF, Ingelsson E. Subfertility and risk of later life maternal cardiovascular disease. Hum Reprod 2012;27:568-75.

14. Sotiriadis A, Makrigiannakis A, Stefos T, Paraskevaidis E, Kalantaridou SN. Fibrinolytic defects and recurrent miscarriage: a systematic review and meta-analysis. Obstet Gynecol 2007;109:1146-55.

15. Andrews FM, Abbey A, Halman LJ. Stress from infertility, marriage factors, and subjective well-being of wives and husbands. J Health Soc Behav 1991;32:238-53.

16. Agarwal A, Aponte-Mellado A, Premkumar BJ, Shaman A, Gupta $S$. The effects of oxidative stress on female reproduction: a review. Reprod Biol Endocrinol 2012;10:49.

17. Prevention of diabetes mellitus: report of a WHO study group. World Health Organ Tech Rep Ser 1994;844:1-100.

18. Lawlor DA, Emberson JR, Ebrahim S, Whincup PH, Wannamethee SG, Walker $\mathrm{M}$, et al. Is the association between parity and coronary heart disease due to biological effects of pregnancy or adverse lifestyle risk factors associated with child-rearing? Findings from the British Women's Heart and Health Study and the British Regional Heart Study. Circulation 2003;107:1260-4.

19. Parikh Nl, Cnattingius S, Dickman PW, Mittleman MA, Ludvigsson JF, Ingelsson E. Parity and risk of later-life maternal cardiovascular disease. Am Heart J 2010;159:215-21.e6.

20. Solomon CG, Hu FB, Dunaif A, Rich-Edwards JE, Stampfer MJ, Willett WC, et al. Menstrual cycle irregularity and risk for future cardiovascular disease. J Clin Endocrinol Metab 2002;87:2013-7.

21. Gregg EW, Cheng YJ, Cadwell BL, Imperatore G, Williams DE, Flegal KM, et al. Secular trends in cardiovascular disease risk factors according to body mass index in US adults. JAMA 2005;293:186874.

22. Flynn RW, Macdonald TM, Jung RT, Morris AD, Leese GP. Mortality and vascular outcomes in patients treated for thyroid dysfunction. J Clin Endocrinol Metab 2006;91:2159-64.

23. Kurabayashi T, Mizunuma H, Kubota T, Hayashi K. Ovarian infertility is associated with cardiovascular disease risk factors in later life: a Japanese cross-sectional study. Maturitas 2016;83:33-9.

24. Reindollar RH. Contemporary issues for spontaneous abortion. Does recurrent abortion exist? Obstet Gynecol Clin North Am 2000;27:541-54.
25. Irgens HU, Reisaeter L, Irgens LM, Lie RT. Long term mortality of mothers and fathers after pre-eclampsia: population based cohort study. BMJ 2001;323:1213-7.

26. Della-Morte D, Beecham A, Dong C, Wang L, McClendon MS, Gardener $\mathrm{H}$, et al. Association between variations in coagulation system genes and carotid plaque. J Neurol Sci 2012;323:93-8.

27. Verit FF, Erel O, Celik N. Serum paraoxonase-1 activity in women with endometriosis and its relationship with the stage of the disease. Hum Reprod 2008;23:100-4.

28. Kinugasa S, Shinohara K, Wakatsuki A. Increased asymmetric dimethylarginine and enhanced inflammation are associated with impaired vascular reactivity in women with endometriosis. Atherosclerosis 2011:219:784-8.

29. Santoro L, D'Onofrio F, Campo S, Ferraro PM, Tondi P, Campo V, et al. Endothelial dysfunction but not increased carotid intima-media thickness in young European women with endometriosis. Hum Reprod 2012;27:1320-6.

30. Wellons M. Cardiovascular disease and primary ovarian insufficiency. Semin Reprod Med 2011;29:328-41.

31. Verit FF, Keskin S, Omer B, Yalcinkaya S, Sakar N. Is there any relationship between cardiovascular risk markers and young women with diminished ovarian reserve? Gynecol Endocrinol 2014; 30:697-700.

32. Purcell SH, Moley KH. The impact of obesity on egg quality. J Assist Reprod Genet 2011;28:517-24.

33. Michalakis K, Coppack SW. Primary ovarian insufficiency: relation to changes in body composition and adiposity. Maturitas 2012;71:320-5.

34. Pacchiarotti A, Mohamed MA, Micara G, Linari A, Tranquilli D, Espinola $\mathrm{SB}$, et al. The possible role of hyperhomocysteinemia on IVF outcome. J Assist Reprod Genet 2007;24:459-62.

35. Gaikwad S, Garrido N, Cobo A, Pellicer A, Remohi J. Bed rest after embryo transfer negatively affects in vitro fertilization: a randomized controlled clinical trial. Fertil Steril 2013;100:729-35.

36. Loftis JM, Huckans M, Morasco BJ. Neuroimmune mechanisms of cytokine-induced depression: current theories and novel treatment strategies. Neurobiol Dis 2010;37:519-33.

37. Khovidhunkit W, Kim MS, Memon RA, Shigenaga JK, Moser AH, Feingold $K R$, et al. Effects of infection and inflammation on lipid and lipoprotein metabolism: mechanisms and consequences to the host. J Lipid Res 2004;45:1169-96.

38. Rizzo M, Rizvi AA, Rini GB, Berneis K. The therapeutic modulation of atherogenic dyslipidemia and inflammatory markers in the metabolic syndrome: what is the clinical relevance? Acta Diabetol 2009;46:1-11. 